\title{
Representation and Communication in Information Systems - A Speech Act Based Approach
}

\author{
Paul Johannesson \\ Department of Computer and Systems Sciences \\ Stockholm University \\ Electrum 230, S-164 40 Kista, Sweden \\ email: pajo@sisu.se
}

\begin{abstract}
One of an information system's important roles is to provide a representation of a Universe of Discourse, which reflects its structure and behaviour. An equally important function of the system is to support communication within an organisation by structuring and coordinating the actions performed by the organisation's agents. In many systems development methods, these different roles that an information system assumes are not explicitly separated. Representation techniques appropriate for one role are uncritically applied to another. In this paper, we propose a unifying framework based on speech act theory, which reconciliates the representation and communication roles of information systems. In particular, we show how communication can be modelled by means of discourses, which are viewed as sequences of events.
\end{abstract}

\section{Introduction}

There are several different views of the functional role of information systems. Two of the most important are the model view and the communicative action view. According to the model view, the primary purpose of an information system is to provide a model of a Universe of Discourse (UoD), thereby enabling people to obtain information about reality by studying the model. In this respect, an information system works as a passive repository of data that reflects the structure and behaviour of the UoD. In contrast, the communicative action view states that the major role of an information system is to support communication within an organisation by structuring and coordinating the actions performed by the organisation's agents. The system is seen as a medium through which people can perform social actions, such as stating facts, making promises, and giving orders. In certain cases, the system can itself take on the role of an agent and perform actions on its own initiative.

Most representation techniques used in systems development are based on the model view of information systems. For example, Entity-Relationship diagrams are used to represent the static and structural aspects of a UoD. Further examples are entity life cycle diagrams and Jackson Structure diagrams, which describe the behaviour of objects. Another technique, which focuses more on communicative aspects, is the data flow diagram technique by which the information and control flow between agents in an organisation can be represented.

The aim of this paper is to show how the model view and communicative action view of information systems can be reconciliated. For this purpose, we propose a speech act based formalism for describing the structure of communicative action. The graphical 
representation of this formalism is very similar to data flow diagrams, but in contrast to these it also provides a clear connection with the structural object representation of a UoD, as expressed in a conceptual schema. In the next section, we briefly review the data flow diagram technique and indicate some of its shortcomings when representing communicative action. In section 3 , we give an outline of speech act theory. In section 4 , we focus on the model view of information systems and present a logic based approach to conceptual modelling. The main results of the paper are given in section 5 , where we introduce a formalism for representing communicative action. In the final section, we summarise the paper and suggest some possibilities for further research.

\section{Data Flow Diagrams}

When trying to understand the information requirements of an organisation, it becomes necessary to conceptualise the way data moves through the enterprise, the processes or transformations that the data undergoes, and what the outputs are. For these purposes, data flow diagrams are used to graphically characterise data processes and flows in an organisation. The data flow diagram is fundamental to structured systems methodologies, such as STRADIS, [Gane79], and was developed as an integrated part of these methodologies. There are four basic concepts in the data flow diagram technique: the data flow, the process, the external agent, and the data store.

One of the most important features of the data flow diagram technique is the ability to construct a variety of levels of data flow diagrams according to the level of abstraction required. An overview diagram can be consulted in order to obtain a high level understanding of a system. Any part of this overview diagram can then be exploded and examined at a more detailed level. The different levels of diagrams must be consistent with each other so that data flows present on the higher levels also exist on the lower levels. In essence it is the processes which are expanded at a greater level of detail as we move down through the levels of a diagram. This hierarchical decomposition process gives the data flow diagram technique its top-down characteristics.

The data flow diagram technique has received wide-spread acceptance and is now an important part of several systems development methodologies. However, the technique has also been heavily criticised, see for example [Buben88], [Coad90], [Auramäki88], [Opdah193]. One line of critique argues that the basic concepts of data flow diagrams are inappropriate for describing the activities of an organisation. When using data flow diagrams, an enterprise is viewed as a collection of physical places or objects, and the work performed is accordingly analysed as physical activities such as sending and storing data. This focus on the physical transfer of data results in a computer and technology biased representation of the communication taking place in an organisation. Thus, the very starting point of the data flow diagram technique is itself flawed and should be replaced by an approach that focuses on the communicative actions of an enterprise.

Another critique pertains to the hierarchical decomposition of data flow diagrams. One problem here is that of knowing how to decompose a diagram and in particular when to stop the decomposition process. A practical rule of thumb is that the decomposition should end before the diagram becomes too cluttered. However, with the arrival of CASE tools and their ability to suppress details this answer is not especially helpful. Another objection to the hierarchical decomposition approach is that the modelling of complex interaction and behaviour cannot be carried out in a top-down fashion. In general, it is not clear at the top stages of decomposition what kinds of activities to include in a process. Instead, the analysis process has to proceed in a combined bottom-up and top-down fashion. Still another problem is that the relationship between data flow diagrams and semantic data models is not clear, although some work on this issue has appeared during 
the last years, [Kung89], [Batini91]. An important aim of this paper is to show how the critique outlined above can be answered. We do this by providing a bridge between semantic data models and data flow diagrams and by reinterpreting the concept of a process using speech act theory.

\section{Speech Act Theory}

An important feature of language is that it can serve purposes other than that of representing the states of affairs of the world. Certain statements are equivalent to actions. For example, when someone says "I apologise", "I promise...", or "I name this ship...", the utterance immediately conveys a new psychological or social reality. An apology takes place only when someone admits having been at fault, and a ship is named only when the act of naming is complete. In such cases, to speak is to perform. Statements such as those above are called performatives or speech acts, and they make it possible to use language as a means both for acting and for coordinating action. The study of speech acts has been an active research area in analytical philosophy since World War II, and the most influential approach to date is speech act theory as developed by John Searle, [Searle69], [Searle86]. Searle proposes a taxonomy for speech acts consisting of five classes: assertives, commissives, directives, declaratives, and expressives. These are also called the illocutionary points of a speech act.

An assertive is a speech act, the purpose of which is to convey information about some state of affairs of the world from one agent, the speaker, to another, the hearer. Examples of assertives are "It is raining" and "The cat is on the mat". A commissive is a speech act, the purpose of which is to commit the speaker to carry out some action or to bring about some state of affairs. Examples of commissives are "I promise to be at home before nine o'clock" and "I swear to bring it back". A directive is a speech act, where the speaker requests the hearer to carry out some action or to bring about some state of affairs. Examples of directives are "Please bring me the salt" and "I order you to leave the room". A declarative is a speech act, where the speaker brings about some state of affairs by the mere performance of the speech act. Examples of declaratives are "I hereby pronounce you man and wife" and "I hereby baptise you to Samuel". An expressive is a speech act, the purpose of which is to express the speaker's attitude about some state of affairs.

Searle makes a distinction between elementary and complex speech acts. An elementary speech act has the form $F(P)$, where $F$ is the illocutionary point and $P$ is the propositional content of the act. Different speech acts may have the same propositional content but different illocutionary points. For example, the assertive "You are going to leave the room" and the directive "I order you to leave the room" both have the same propositional content, but their illocutionary points differ. There are also complex speech acts of which the two most important forms are the denegated speech act and the conditional speech act. A denegated speech act has the form $\neg \mathrm{F}(\mathrm{P})$. This expresses that the speaker is not prepared to perform the speech act F(P); for example "I do not promise to come". A conditional speech act has the form $p \rightarrow F(P)$. This expresses that the speaker will perform the speech act if some condition is fulfilled; for example "If it rains, I will go to the cinema with you".

Most speech acts can only be successful if certain conditions hold. For example, a promise is successfully made only if the speaker is able to carry out the promised act and this act is in the interest of the hearer. Similarly if a speaker apologises he presupposes that the thing he apologises for is bad or reprehensible. Conditions that in this way are necessary for the performance of a speech act are called preparatory conditions. They can be thought of as certain sorts of states of affairs that have to prevail in order for a speech act to be successful. 
There are a few contributions in the literature on the application of speech act theory to information systems. Flores et. al. have developed a speech act based tool, the Coordinator, which is used to coordinate communicative action within an organisation in the context of collaborative work, [Flores88]. The Coordinator is primarily intended to support semiformal and informal communication. Auramakki, Lehtinen, and Lyytinen have proposed a modelling approach based on speech acts called SAMPO, which studies office activities as a series of speech acts creating, maintaining, modifying, reporting, and terminating commitments, [Auramakki88]. They introduce the concept of a discourse, as a sequence of speech acts, and suggest some graphical notations for representing discourse types. Dietz has proposed a novel approach to process modelling based on speech acts, called the Essential Process Model, in which essential business activities are clearly separated from non-essential, i.e., informative, activities, [Dietz92]. The approach presented in this paper is in many respects similar to that of SAMPO. One difference, however, is that we attempt to provide a clear connection between models of communicative action and conceptual schemas representing the structure and behaviour of a UoD. Further, we do not propose any completely new graphical notation for discourse types. Instead, we try to keep the notation as close as possible to ordinary data flow diagrams and show how the constructs of data flow diagrams can be reinterpreted using speech act theory.

\section{Conceptual Schemas}

In this section, we attempt a formalisation of some of the basic notions in conceptual modelling. We first recall some definitions conceming first order languages. Let $P r$ and $C$ be two sets of symbols. A (first order) language based on $\langle\operatorname{Pr}, C\rangle$, written $L(P r, C)$ is defined on an alphabet consisting of connectives, quantifiers, punctuation symbols, variables, constants $C$, and predicate symbols $P r$, where each predicate symbol has an arity. A term is a constant or a variable. A (first order) formula in a language $L$ is defined as usual. An atom is a formula of the form $p\left(t_{1}, \ldots, t_{n}\right)$, where $p$ is a predicate symbol and $t_{1}, \ldots, t_{n}$ are terms. A literal is an atom or a negated atom. A ground formula is a formula without variables. For any language $L(\operatorname{Pr}, C)$ we assume that Pr contains a special symbol, "=", which is interpreted as the identity.

We now introduce some notions for describing the behaviour of a UoD. These will be based on the assumption that the dynamics of an application can be modelled through events which are grouped together in event types.

Definition 4.1: An update operation is a formula of one of the following forms: - insert(L), where $L$ is a positive literal - delete( $(\mathrm{L})$, where $\mathrm{L}$ is a positive literal

A ground update operation is an update operation, in which all terms are ground. A symbol generator is a formula of the form "new(S)", where $S$ is a variable.

Definition 4.2: An event type is a triple $<\mathrm{P}$, Pre, Post $\rangle$, where

$P$ is a set of variables, called the input variables of the event type. Pre is a conjunction of literals. Post is a list of update operations and symbol generators, and the variables in Post constitute a subset of those in $\mathrm{P}$ and Pre. Pre is called the preconditions of the event type and Post the post conditions. We shall sometimes use the following notation for event types: "if Pre then Post", leaving P implicit. 
A fundamental notion in conceptual modelling is that of the conceptual schema, which is usually informally defined as an implementation independent description of the contents in an information system.

Definition 4.3: A conceptual schema is a triple $<\mathrm{L}, \mathrm{IC}, \mathrm{ET}\rangle$, where $\mathrm{L}$ is a language, IC is a finite set of closed formulas, called integrity constraints, and ET is a set of event types. It is assumed that all predicate symbols in L are either unary or binary, and that for each binary predicate symbol $p$ there is an integrity constraint in the form:

$\forall \mathrm{x} \forall \mathrm{y}(\mathrm{p}(\mathrm{x}, \mathrm{y}) \rightarrow \mathrm{p} 1(\mathrm{x}) \wedge \mathrm{p} 2(\mathrm{y}))$. $\mathrm{p} 1$ is called the domain of $\mathrm{p}$, and $\mathrm{p} 2$ the range of $\mathrm{p}$.

The unary predicate symbols are called object types and the binary ones are called attributes.

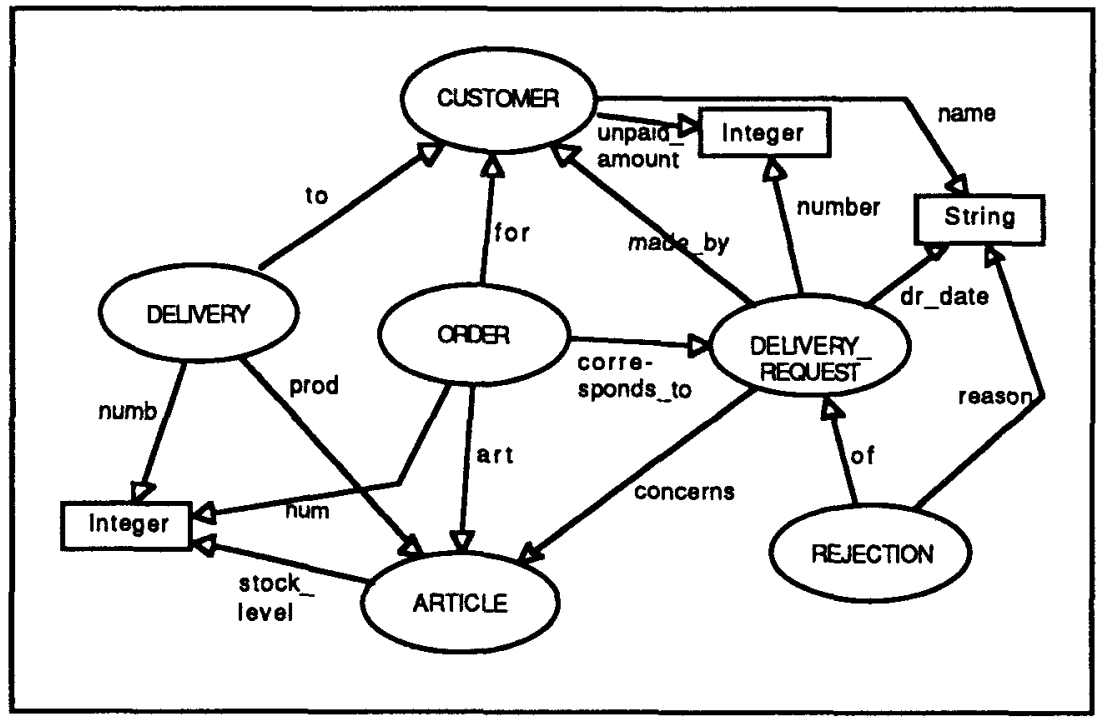

Fig. 4.1 Example of a Conceptual Schema

Example 4.1: In fig. 4.1, a graphical representation of a conceptual schema is shown. Note that the graph only depicts a part of the language of the schema and some integrity constraints. The graph shows that the schema contains eight object types \{customer, delivery, delivery_request, rejection, order, article, integer, string $\}$ and sixteen attributes, displayed as labeled arcs. The graph also shows a number of integrity constraints, e.g., $\forall x \forall y($ made_by $(x, y) \rightarrow$ delivery_request $(x) \wedge$ customer $(y))$. Object types containing data values are shown in the graph as rectangles, in this case "String" and "Integer".

Example 4.2: Continuing example 4.1, we give three examples of event types, which will be used throughout this paper. We use the convention of beginning variables with a capital letter.

make_delivery_request:

$<$ \{Customer, Article, Number, Date\},

$\varnothing$,

[new(DR), insert(delivery_request(DR)), insert(made_by(DR, Customer)), insert(concerns(DR, Article)), insert(number(DR, Number)), insert(dr_date(DR, Date))]> 
make_onder:

$<$ Customer, Article, Number, Del_request $\}$, (stock_level(Article, $S$ ) $\wedge S>$ Number, unpaid_amount(Customer,Up) $\wedge$ Up $<50000$ ) [new(O), insert(order(O)), insert(corresponds_to(O, Del_request)), insert(art(O, Article)), insert(num( $O$, Number $)$ ), insert(for $(O$, Customer) $)]>$

rejection:

$<$ Del_request, Reason>, $\varnothing$, [new(R), insert(rejection(R)), insert(of(R, Del_request)), insert(reason(R, Reason)).

We now turn to the definition of an information base; informally an information base contains information about particular entities and the associations between these.

Definition 4.4: An information base for a Conceptual Schema $<$ L, IC, ET $>$ is a pair $\langle C, F\rangle$, where $\mathrm{C}$ is a finite set of constants, and $\mathrm{F}$ is a finite set of ground atoms.

Example 4.3: An example of an information base for the schema in Fig. 4.1 is: customer(c1) name(c1,'Jones') customer(c2) name(c2,'Smith') delivery(d1) to(d1,c1) numb(d1,100) article(a1) $\quad$ prod(d1,a1) $\quad$ stock_level(a1,5000)

The role of integrity constraints is to state conditions that must hold for each information base.

Definition 4.5: Let $\mathrm{CS}=\langle\mathrm{L}, \mathrm{IC}, \mathrm{ET}\rangle$ be a conceptual schema and IB $=\langle\mathrm{C}, \mathrm{F}\rangle$ an information base for CS. The information base IB violates the schema CS if not (FI= IC).

Now we can give the semantics for event types. Intuitively, the idea is that when an event occurs in the UoD, it will be reported by submitting an event message. Based on the event message, the event type, and the present information base, a new information base will be computed. First, the preconditions are checked, and if they are satisfied in the present information base, a new information base is derived by applying the post conditions. If the preconditions are not satisfied, no updates will be performed. Informally, the semantics of the predicate symbol "new" is to generate a new constant that has not been used before.

Definition 4.6: An event occurrence is a pair $\langle E, S>$ where $E=\langle P$, Pre, Pos $>$ is an event type and $S$ is a ground substitution that binds each variable in $P$.

Definition 4.7: A transaction is a formula of the form

if $\alpha_{1}, \ldots, \alpha_{n}$ then $p_{1} ; \ldots ; p_{m}$,

where the $\alpha_{i s}$ are ground literals and the pis are ground update operations or symbol generators. A transaction is expressible in a language $\mathrm{L}$ if all literals in the transaction are expressed in $\mathrm{L}$. 
Definition 4.8: Let $C S=\langle L, I C$, ET $\rangle$ be a conceptual schema. We define a function $\mu$ : $\mathrm{IBCS}^{\times} \mathrm{TCS} \rightarrow \mathrm{IBCS}$, where IBCS is the set of all information bases for CS and TCS is the set of all transactions expressible in $\mathrm{L}$.

Let $\mathrm{IB}=\langle\mathrm{C}, \mathrm{F}\rangle$. We define $\mu$ recursively:

$\mu\left(\mathrm{IB}\right.$, if $\alpha_{1}, \ldots, \alpha_{\mathrm{n}}$ then $\left.\mathrm{p} 1\right)=\mathrm{IB}$ if $\operatorname{not}\left(\mathrm{F} \mid=\alpha_{1} \wedge \ldots \wedge \alpha_{\mathbf{n}}\right)$

$=\mathrm{IB}^{\prime}$ if $\mathrm{F} \mid=\alpha_{1} \wedge \ldots \wedge \alpha_{\mathrm{n}}$, where

$\mathrm{IB}^{\prime}=\left\langle\mathrm{CU}\left\{\mathrm{A}_{\mathrm{C}}\right\}, \mathrm{FU}\{\mathrm{A}\}>\right.$ if $\mathrm{p}_{1}=\operatorname{insert}(\mathrm{A})$

$\mathrm{IB}^{\prime}=\langle\mathrm{C}, \mathrm{F}-\{\mathrm{A}\}\rangle$ if $\mathrm{p}_{1}=\operatorname{delete}(\mathrm{A})$

$\mathrm{IB}^{\prime}=\mathrm{IB}$ if $\mathrm{p} 1=$ new(S)

( $A C$ is the set of constants appearing in the formula $A$.)

$\mu\left(\mathrm{IB}\right.$, if $\alpha_{1}, \ldots, \alpha_{\mathrm{n}}$ then $\left.\mathrm{p}_{1} ; \ldots ; \mathrm{pm}\right)=\mathrm{IB}$ if not $\left(\mathrm{F} \mid=\alpha_{1} \wedge \ldots \wedge \alpha_{\mathrm{n}}\right)$

$=\mu\left(\mathrm{IB}^{\prime}\right.$, if true then $\left.\mathrm{p} 2 ; \ldots ; \mathrm{p}_{\mathrm{m}}\right)$

otherwise, where IB' is defined as above.

Definition 4.9: Let $\mathrm{CS}=\langle\mathrm{L}$, IC, ET $>$ be a conceptual schema. Let IBCS be the set of all information bases for CS, and EO the set of all event occurrences for event types in ET.

Define a function PerformCS: $\mathrm{EO} \times \mathrm{IBCS}_{\mathrm{C}} \rightarrow \mathrm{IB} \mathrm{CS}$, by

$(<<$ P,Pre,Pos $\downarrow, \theta>, I B) \mapsto \mu(I B$, if $\operatorname{Pre}[\Psi]$ then Post $[\Psi])$, if there exists a unique extension $\Psi$ of $\Theta$ such that $F \mid=\operatorname{Pre}[\Psi],(I B=\langle C, F\rangle)$

$\rightarrow$ IB, otherwise

The approach to modelling dynamics that we have chosen has certain limitations compared to some of the more advanced approaches in the literature, such as that of LDL, [Naqvi89]. For example, in definition 4.9 , we require the existence of a unique extension of the incoming event message, while other approaches may accept several extensions. Further, we do not attempt to formalise the behavioural aspects of a conceptual schema within the framework of a logic formalism, such as dynamic logic. However, the approach above has the advantage of capturing our intuitions about the event concept in a simple way, and it is sufficient to serve as a vehicle for analysing the relationship between semantic data models and communicative action modelling.

Example 4.4: Let CS be the conceptual schema from Fig 4.1, let IB be the information base in example 4.3, and let Eo be the event occurrence <make_delivery_request, \{Customer/c1, Article/al, Number/25, Date/930601\}).

Then, Perform CS(Eo,IB) is the following information base:

$\begin{array}{llll}\text { customer(c1) } & \text { name(c1,'Jones') } & \text { customer(c2) } & \text { name(c2,'Smith') } \\ \text { delivery(d1) } & \text { to(d1,c1) } & \text { numb(d1,100) article(a1) } \\ \text { prod(d1,a1) } & \text { stock_level(a1,5000) } & \text { delivery_request(dr1). } \\ \text { made_by(dr1,c1) } & \text { concems(dr1,a1) } & \text { number(dr1,25) dr_date(d1,930601) }\end{array}$

By means of the event concept it is possible to describe the dynamics of a UoD. However, describing the dynamics using only event types has the limitation that the interrelationships among various event types are not made explicit. There is no way of specifying that certain events must precede or usually precede other events, or that the occurrence of certain events excludes the occurrence of others, etc. To represent these types of interrelationships among events, we need to put an additional structure on top of the 
event types. In the next section, we propose such a structure for the special case of events corresponding to communicative actions.

\section{Discourse Structures}

We will now present a speech act based formalism for the description of control and information flow in communicative action. Our point of departure is that the event types in the conceptual schema that correspond to communicative actions should be organised by so called discourse structures. A discourse structure is a graph that displays the form of a dialogue, or discourse, between two or more agents. We make the assumption that for each type of discourse, there is a unique organisational unit (department, person etc.) responsible for that type of discourse. The basic concept of the formalism is that of a speech act, formally defined as below.

Definition 5.1: Let CS(L, IC, ET) be a conceptual schema. A speech act is a quintuple $<$ IIlPoint, Speaker, Hearer, PrepCond, PropCont>, where Illpoint $E$ \{assertive, commissive, directive, declarative \}, Speaker and Hearer are either object types in CS or the special symbol "unit", PrepCond is a set of formulas in $\mathrm{L}$, and PropCont is a formula in L. A speech move is a set of speech acts.

In definition 5.1, we require that the hearer and speaker of a speech act are explicitly modelled as types in the conceptual schema, except for the case where the speaker or hearer is the organizational unit responsible for the discourse in which the speech act takes place - in the latter case the symbol "unit" is used. This requirement is a deviation from current practice in conceptual modelling, where the agents in the environment of an information system are often omitted from the conceptual schema. However, including these agents in the schema is an important means of relating the conceptual schema to the modelling of communicative action.

The component PrepCond in the definition of a speech act specifies the preparatory conditions, and PropCont the propositional content. The formula PropCont is to describe some state of affairs. In the case of an assertion, the speaker asserts that this state of affairs prevails. In the case of a commissive, he commits himself to bring about this state of affairs. In the case of a directive, he requests another agent to bring it about, and in the case of a declarative, he brings it about simply by performing the speech act. In the graphical notation we are going to use, a speech act is displayed as a rounded box with six compartments, as shown in fig. 5.1. The meaning of the contents in the top compartment is explained below; the other compartments correspond to the five components of a speech act. In the example of fig. 5.1, the speaker requests the hearer to create a situation where a certain delivery to the hearer has been performed.

We also introduce the concept of a speech move. The motivation for this concept is that certain speech acts are always performed simultaneously and therefore constitute a natural unit, or move, in a discourse. An example of a speech move is the borrowing of a book at a library, where two speech acts are performed at the same time. First, the library performs a declarative, allowing the borrower to have the book at his disposal for a certain period of time. Secondly, the borrower performs a commissive, promising to return the book within a certain time limit.

We now turn to the question of how to represent the structure of discourses. An adequate representation must provide the means of expressing both the control flow and the information flow between the speech moves of a discourse. When exchanging speech acts, the agents can often choose between several alternatives at each particular point of the discourse. Consequently, it is necessary to be able to model the control flow and the 
rules that govern the choices being made by the agents. Further, objects introduced by one agent in a certain speech move may be referred to by the same or another agent in subsequent speech moves of the discourse. Thus, it must be possible to represent the flow of information between speech moves. In the following definition, we introduce the concept of a discourse structure.

\begin{tabular}{|c|c|c|}
\hline \multicolumn{3}{|c|}{$\begin{array}{l}\text { make_delivery_request: } \\
\text { Customer, Article, Number, Date + DR }\end{array}$} \\
\hline $\begin{array}{l}\text { ILLPOINT } \\
\text { directive }\end{array}$ & $\begin{array}{l}\text { SPEAKER } \\
\text { customer }\end{array}$ & $\begin{array}{l}\text { HEARER } \\
\text { unit }\end{array}$ \\
\hline \multicolumn{3}{|c|}{ PREPARATORY CONDITIONS } \\
\hline \multicolumn{3}{|c|}{$\begin{array}{l}\text { PROPOSITIONAL CONTENT } \\
\text { delivery(D) } \wedge \text { to(D, Customer }) \wedge \\
\text { numb(D, Number }) \wedge \text { prod(D, Article })\end{array}$} \\
\hline
\end{tabular}

Fig 5.1 Example of a speech act

Definition 5.2: A control statement is a closed formula.

Definition 5.3: Let $\mathrm{CS}=\langle\mathrm{L}, \mathrm{IC}, \mathrm{E}\rangle$ be a conceptual schema. A discourse structure is a quadruple $\langle D, V, \phi, \psi\rangle$, where $D$ is a directed graph $\langle N, A\rangle$, and $N=S M U C T$ is a set of speech moves and control statements, and $A$ is a set of arcs. $V: A \rightarrow(2 \operatorname{Var} U$ \{true, false\}) is a function, where Var is the set of variables in $L . \phi: S M \rightarrow E$ is an injective function. $\%: S M \rightarrow T$ is an injective function, where $T$ are the types in $\mathrm{L}$. A discourse structure must satisfy the following conditions:

(i) For each $s$ in SM, $\phi(s)$ includes the postconditions "new(Y), insert $(\psi(s)(Y))$ "

(ii) For each $s$ in $S M$, pre $(\phi(s))=U$ (prep(sa) $\mid s a \in s\}$, where prep and pre are functions returning the preparatory conditions of a speech act, and the preconditions of an event, respectively

(iii) For any arc a from $n_{1}$ to $n_{2}, V(a) \subset \operatorname{Var}\left(\phi\left(n_{1}\right)\right)$ if $n_{1} \in S M$, and $V(a) \subset \operatorname{Arg}\left(\phi\left(n_{2}\right)\right)$ if $\mathrm{n}_{2} \in \mathrm{SM}$, where Var is a function returning the set of all variables in an event type, and Arg is a function returning the input variables of an event type

(iv) For any $c$ in CT, there is a path from $c$ to an $s$ in SM such that $s$ contains an sa with ce prep(sa)

(v) For each c in CT, there are exactly two arcs leaving c, one of them labelled "true", the other "false"

A basic discourse structure is a discourse structure, where all nodes are speech moves.

The functions $\phi$ and $\psi$ specify the relationships between a conceptual schema and a discourse structure. For each speech move, there must exist a unique corresponding object type in the conceptual schema - this is given by the function $\psi$. Thus, all speech moves must be explicitly modelled in the schema. Similarly, for each speech move there must exist a unique corresponding event type - this is given by $\phi$. Further, condition (i) states that at each occurrence of this event type, an object corresponding to the speech move must be created. In this way, a record is kept of all speech moves occurring. Note that the 
functions $\phi$ and $\psi$ are not surjective; there may well exist event types and object types that do not correspond to any speech move. Condition (ii) requires that the preparatory conditions of a speech move be equal to the preconditions of the corresponding event type. The information flow in a discourse is expressed by means of the variables associated with the arcs, and condition (iii) states that a variable may occur on an arc only if it has been introduced in one speech move and is later to be used in another. The control flow in a discourse is represented by the control statements of a discourse structure. Condition (iv) places a restriction on which control statements may occur in a discourse structure: each control statement must be equal to a preparatory condition in a subsequent speech move. Informally, the purpose of condition ( $v$ ) is to express that if a control statement is true, then the discourse is to continue along the arc labelled "true" and otherwise along the arc labelled "false". In the graphical notation, see fig. 5.1, the topmost compartment contains the name of the event type corresponding to the speech move and its input variables. Further, the new variable referred to in condition (i) is specified after the " + " sign, thereby providing a handle to the speech move.

When constructing a discourse structure, one possible starting point is to construct a basic discourse structure containing only speech moves that shows the basic and typical form of the discourse. This discourse structure can then be expanded by adding control statements corresponding to preparatory conditions in the speech moves and specifying which actions to undertake when a preparatory condition is not satisfied. In this way, a discourse structure can be systematically expanded until no more control statements can be added. In the definition below, we introduce the term "extended discourse structure" to denote a discourse structure that cannot be extended any further.

Definition 5.4: An extended discourse structure $\langle\mathrm{D}, \mathrm{V}, \phi, \psi\rangle$, where $\mathrm{D}=\langle\mathrm{SM} U \mathrm{CT}$, $A>$ is a discourse structure where for each preparatory condition $P$ occurring in some speech act in SM, there exists a $\mathrm{c} \in \mathrm{CT}$ such that $\mathrm{P}=\mathrm{c}$, and there is a path from $\mathrm{c}$ to the speech move in which $P$ occurs.

In fig. 5.2, a basic discourse structure is shown. The first speech act is the same as the one in fig. 5.1, whereas the second is a promise by the speaker to deliver an article to the hearer, thereby satisfying the request of the preceding speech act. This discourse structure is extended in fig. 5.3 by including two control statements, which check whether the stock level is sufficient and that the customer's debt is not too large. If these conditions are not satisfied, the customer's request is rejected and an assertive is performed telling the customer why.

We are now in a position to define the term "discourse" precisely as a sequence of speech move occurrences that satisfy certain conditions.

Definition 5.5: Let $\mathrm{DS}=\langle\mathrm{D}, \mathrm{V}, \phi, \psi\rangle$ be a discourse structure. A discourse according to $D S$ is a sequence of event occurrences $\left\langle\mathrm{e}_{1}, \mathrm{sl}\right\rangle, \ldots,\left\langle\mathrm{e}_{\mathrm{n}}, \mathrm{s}_{\mathrm{n}}\right\rangle$ such that there exists a path through $D$ containing a subsequence of speech moves $m_{1}, \ldots, m_{n}$, where $\phi\left(m_{i}\right)=e_{i}$, and if the same variable occurs in two substitutions $s_{i}$ and $s_{j}, i \neq j$, and on some arc of the path, it must be bound to the same constant in each substitution.

Definition 5.6: Let DS be a discourse structure and $D=\left\langle e_{1}, s 1\right\rangle, \ldots,\left\langle e_{n}, s_{n}\right\rangle$ a discourse according to DS. Let I be a non-violating information base. D is successful in I if 
(i) Perform $\left(\left\langle\mathrm{e}_{\mathrm{i}}, \mathrm{s}_{\mathrm{i}}\right\rangle, \mathrm{B}_{\mathrm{i}}\right)$ does not violate $\mathrm{CS}$ for $\mathrm{i}=1, \ldots, \mathrm{n}$, where $\mathrm{B}_{\mathrm{i}}=\operatorname{Perform}\left(<\mathrm{e}_{\mathrm{i}-1}, \mathrm{~s}_{\mathrm{i}}-\right.$ 1>, $\left.B_{i-1}\right)$ and $B_{0}=I$

(ii) The preconditions of the event type $e_{i}$ are satisfied in Perform( $\left.\left\langle e_{i-1}, s_{i-1}\right\rangle, B_{i-1}\right), i=$ $2, \ldots, n$, and $B_{i}$ is defined as above

\begin{tabular}{|c|c|c|}
\hline \multicolumn{3}{|c|}{$\begin{array}{l}\text { make_delivery_request: } \\
\text { Customer, Article, Number, Date + DR }\end{array}$} \\
\hline $\begin{array}{l}\text { IILPOINT } \\
\text { directive }\end{array}$ & $\begin{array}{l}\text { SPEAKER } \\
\text { customer }\end{array}$ & $\begin{array}{l}\text { HEARER } \\
\text { unit }\end{array}$ \\
\hline \multicolumn{3}{|c|}{ PREPARATORY CONDITIONS } \\
\hline \multicolumn{3}{|c|}{$\begin{array}{l}\text { PROPOSITIONAL CONTENT } \\
\text { delivery(D) } \wedge \text { to }(D, \text { Customer }) \wedge \\
\text { numb(D, Number }) \wedge \text { prod }(D, \text { Article })\end{array}$} \\
\hline \multicolumn{3}{|c|}{$\begin{array}{l}\text { \{Customer, Article, } \\
\text { Number, Date\} }\end{array}$} \\
\hline \multicolumn{3}{|c|}{$\begin{array}{l}\text { make_order: } \\
\text { Customer, Article, Number, Date + } 0\end{array}$} \\
\hline $\begin{array}{l}\text { ILLPOINT } \\
\text { commissive }\end{array}$ & $\begin{array}{l}\text { SPEAKER } \\
\text { unit }\end{array}$ & $\begin{array}{l}\text { HEARER } \\
\text { customer }\end{array}$ \\
\hline \multicolumn{3}{|c|}{$\begin{array}{l}\text { PREPARATORY CONDITIONS } \\
\text { (stock_level(Article,S) } \wedge \mathrm{S}>\text { Number, } \\
\text { unpaid_amount(Customer, Up) } \wedge \\
U \mathrm{p}<50000 \text { ] }\end{array}$} \\
\hline \multicolumn{3}{|c|}{$\begin{array}{l}\text { PROPOSITIONAL CONTENT } \\
\text { delivery(D) } \wedge \text { to }(D, \text { Customer }) \wedge \\
\text { numb }(D, N u m b e r) \wedge \text { prod(D, Article })\end{array}$} \\
\hline
\end{tabular}

Fig. 5.2 A basic discourse structure

We will now introduce a completeness requirement that should be satisfied by each discourse structure. When an agent has performed a directive, this should be taken care of by the hearer in a subsequent step of the discourse. The directive can be handled in three different ways. First, the hearer can perform a commissive with the same propositional content as the directive, i.e., the hearer promises to do what the speaker requested. Secondly, the hearer can immediately fulfil the request of the speaker by performing a declarative with the same propositional content as the speaker's directive. Thirdly, the hearer can reject the speaker's request, and in that case he should give a reason for the 
rejection. Usually, the reason is that he could not perform an appropriate commissive or declarative because some preparatory condition was not satisfied.

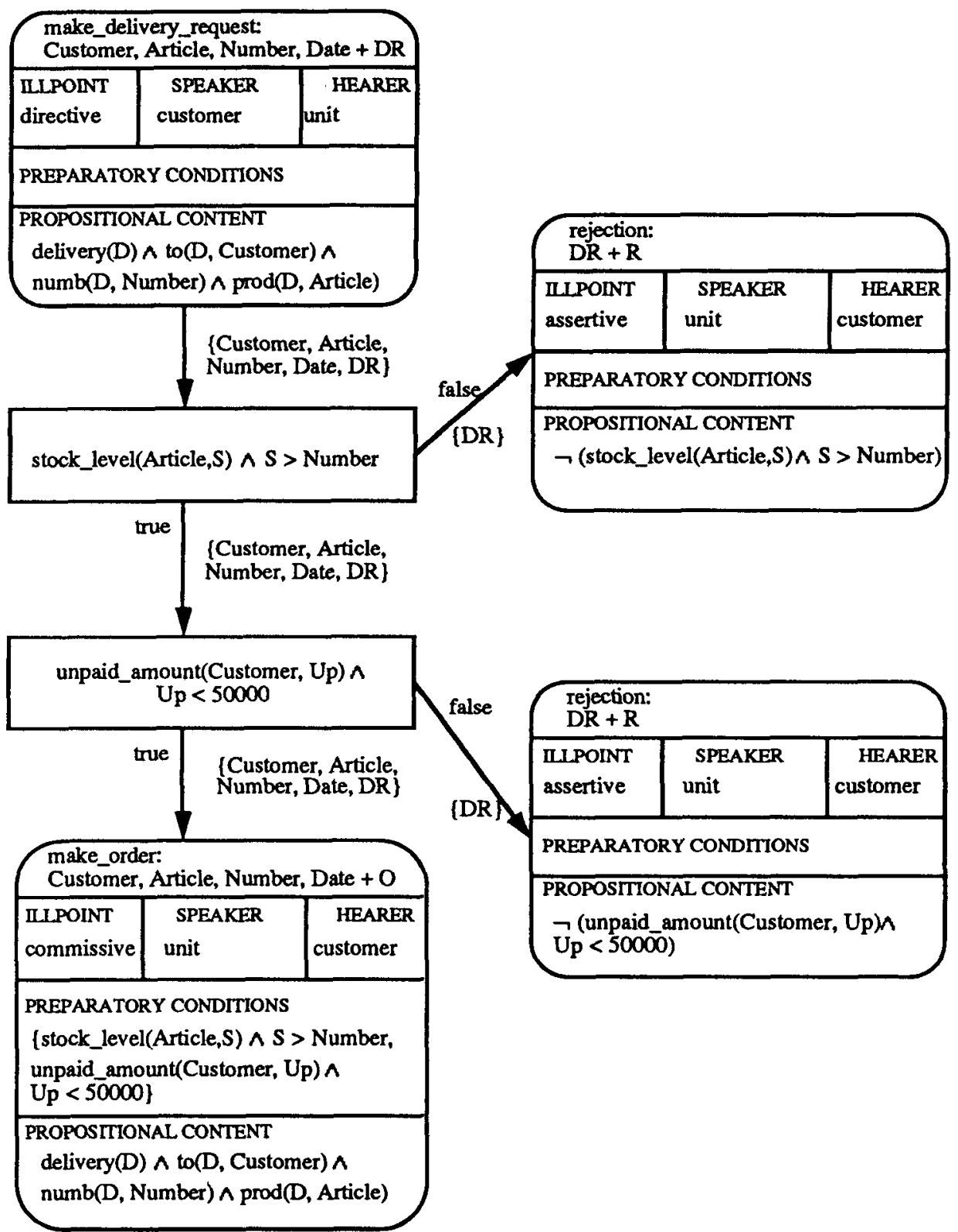

Fig. 5.3 An extended and complete discourse structure 
Definition 5.7: A discourse structure DS is complete if for any speech act $S=$ <directive, Sp, He, Prep, Prop> in DS there exists a speech act T in DS, there is a path from $S$ to $T$, and $T$ has one of the following forms:

(i) $\mathrm{T}=$ <commissive, He, Sp, P, Prop>

(ii) $\mathrm{T}=<$ declarative, $\mathrm{He}, \mathrm{Sp}, \mathrm{P}$, Prop $>$

(iii) $\mathrm{T}=$ <assertive, $\mathrm{He}, \mathrm{Sp}, \mathrm{P},-\mathrm{Pre}$, and there exists a speech act $\mathrm{U}=<$ illpoint, $\mathrm{He}$, Sp, PrepCo, Prop> in DS, Illpoint equals "commissive" or "declarative", and Pre $€$ PrepCo

Note that the discourse structure in fig. 5.3 is complete, since the customer's request is followed either by a corresponding commissive or a rejection.

The completeness requirement introduced above takes into account only a single discourse structure. However, it is relevant to consider a completeness property that spans a set of discourse structures and event types. Informally, the idea is that all directives and commissives should be taken care of in some way. Whenever a directive or commissive is performed, an expectation is created. As an example, when a book is borrowed from a library, the borrower performs a commissive thereby creating an expectation that he is going to return the book within a certain time limit. An expectation created by a speech act in a discourse can either be fulfilled within the same discourse or in another context. In definition 5.7, we required that in a complete discourse structure any directive should be taken care of immediately. A commissive, on the other hand, is usually treated at a later point in time and not in the context of the discourse where it was made. We could then require that an information system should monitor that all expectations created by commissives are fulfilled, and to take appropriate action when they are not. Continuing with the example of the library, the information system should include a function that monitors the return of books and sends out reminders when loans are overdue. It is outside the scope of this paper to formalise this form of completeness. Such a formalisation would require a modelling language including concepts from active databases.

The concepts of completeness in discourse structures are similar to the action workflow protocol, [Medina-Mora92]. In the first phase of an action workflow, the customer asks for a service. In the second phase, the person addressed agrees to carry out the required service. In the third phase, some action is carried out and the service is delivered. Finally, the customer acknowledges the receipt of the service.

\section{Summary and Further Work}

In this paper, we have shown how speech act theory can be used as the basis of a formalism for modelling communicative action in organisations. The formalism we have introduced is very close to the data flow diagram technique but avoids some of its shortcomings. For example, the formalism makes explicit which agents are involved in each action. Further, it provides a guide-line for how to extend discourse structures systematically until all exceptional cases have been taken into account. We have also introduced two completeness requirements for discourse structures, which can be used to validate a design.

The approach of this paper puts constraints on the construction of a conceptual schema by requiring that each agent in the environment of an information system be explicitly modelled as an object type and that each speech move be associated with an object type. By following these requirements, one obtains a conceptual schema that is closely related to the model of the communicative processes. In definition 5.3, we assumed that a conceptual schema should already exist when describing a discourse structure. An interesting observation, however, is that it is possible, almost 
automatically, to construct a conceptual schema from an informally described discourse structure, [Holm93].

The approach presented in this paper represents only a beginning, and much work remains to be done. First, we need to consider also complex speech acts in order to model discourses more accurately. For example, in section 5 we modelled a rejection of a directive as an assertive, but it would have been more correct to represent it as a denegated commissive or declarative. As another example, a request may be answered not only by an acceptance or a rejection, but also by a counteroffer, which should be modelled as a conditional commissive.

Another issue concerns the form of the propositional content of a speech act. In this paper, we have assumed that the propositional content is a formula in the language of a conceptual schema. However, this may be unsatisfactory, since commissives and declaratives are almost invariably associated with some time restriction and this is not reflected in the propositional content. (The most typical time restriction is for some action to be performed by a specified date.) It would therefore be desirable to include a mechanism for representing time in speech acts explicitly. One possible approach here could be to use a temporal conceptual modelling language.

\section{References}

[Auramäki88] E. Auramäki, E. Lehtinen and K. Lyytinen, "A Speech-Act Based Office Modelling Approach", ACM Transactions on Office Information Systems, vol. 6, no. 2, pp. 126 - 152, 1988.

[Batini91] C. Batini, S. Ceri and S. Navathe, Conceptual Database Design, Benjamin/Cummings, 1991.'

[Buben88] J. A. Bubenko Jr., "Problems and Unclear Issues with Hierarchical Business Activity and Data Flow Modelling", SYSLAB Working Paper no. 134, The Royal Institute of Technology and the University of Stockholm, 1988.

[Coad90] E. Coad and D. Yourdon, Object Oriented Analysis and Design, Addison-Wesley, 1990.

[Dietz92] J. Dietz, "Modelling Communication in Organizations", in Linguistic Instruments in Knowledge Engineering, Ed. R. v. d. Riet, pp. 131 - 142, Elsevier Science Publishers, 1992.

[Flores88] F. Flores, M. Graves, B. Hartfield and T. Winograd, "Computer Systems and the Design of Organizational Interaction", ACM Transactions on Office Information Systems, vol. 6, no. 2, pp. 153-172, 1988.

[Gane79] C. Gane and T. Sarson, Structured Systems Analysis: Tools ans Techniques, Prentice-Hall, 1979.

[Holm93] Peter Holm, Swedish Institute of Systems Development, Personal Communication

[Kung89] C. H. Kung, "Conceptual Modeling in the Context of Software Development", IEEE Transactions on Software Engineering, vol. 15, no. 10, pp. 1176 ff., 1989.

[Medina-Mora92] R. Medina-Mora et.al., "The Action Workflow Approach to Workflow management Technology", 4th CSCW Conference, Toronto, 1989.

[Naqvi89] S. Naqvi and S. Tsur, A Logical Language for Data and Knowledge Bases, Computer Science Press, 1989.

[Opdah193] A. Opdahl and G. Sindre, "Concepts for Real-World Modelling, Sth International Conference on Advanced Information Systems Engineering, Ed. C. Rolland, Paris, Springet, 1993.

[Searle69] J. Searte, Speech Acts - An Essay in the Philosophy of Language, Cambrudge University Press, 1969.

[Searle86] J. Searle and D. Vanderveken, Foundations of Illocutionary Logic, Cambridge University Press, 1986. 